\title{
The Gelation Ability and Morphology Study of Organogel System Based on Calamitic Hydrazide Derivatives
}

\author{
Xia Ran, Lili Shi, Kun Zhang, Jie Lou, Bo Liu, and Lijun Guo \\ Institute of Photobiophysics, School of Physics and Electronics, Henan University, Kaifeng 475004, China \\ Correspondence should be addressed to Lijun Guo; juneguo@henu.edu.cn
}

Received 1 August 2014; Accepted 19 September 2014

Academic Editor: Tifeng Jiao

Copyright (c) 2015 Xia Ran et al. This is an open access article distributed under the Creative Commons Attribution License, which permits unrestricted use, distribution, and reproduction in any medium, provided the original work is properly cited.

\begin{abstract}
The gelation property of a series of LMOG bearing hydrazide and azobenzene groups, namely, N-4-(alkoxyphenyl)- $\mathrm{N}^{\prime}-4$-[(4methoxyphenyl)azophenyl] benzohydrazide (BNBC- $n, n=8,12,14$ ), has been systematically studied in this work. The obtained results demonstrate that the gelling ability in organic solvents is significantly influenced by the length of terminal alkoxy chain. In different organic solvents, it is hard to observe the organogel formation for BNBC-8 molecule. On the contrary, the organogelators BNBC-12 and BNBC-14 bearing longer terminal chains have shown great ability to gel organic solvents to form stable organogels. The critical gelation concentration for BNBC-12 reaches as low as $5.3 \times 10^{-3} \mathrm{M}$, which can be considered as a supergelator. It has been manifested that the aggregation morphology of organogel strongly depends on the nature of the gelling solvents and the length of the terminal alkoxy chain. The gelation of BNBC- $n$ provides an easy method for the preparation of multidimensional structure and manipulation of morphology from ribbons, hollow tube fiber to 3D net-like structure in different solvents. The cooperation of hydrogen bonding, $\pi-\pi$ interaction, and Van der Waals force is suggested to be the main contribution to this self-assembled structure.
\end{abstract}

\section{Introduction}

In the past decades, low molecular mass organogelators (LMOGs) have attracted broad attention because they can self-assemble into diverse nano/microstructures, for instance, particles, tubes, fibers, and helical ribbons, through specific noncovalent interactions such as hydrogen bonding, $\pi-\pi$ interaction, hydrophobic interaction and Van der Waals force, and so forth [1-3]. Supramolecular self-assembly is a spontaneous process of molecular aggregation into ordered nanostructures, which provides a bottom-up approach to obtain structural regularity of various morphologies [4]. However, a complete mechanism for the self-assembled supramolecular structures is still beyond our understanding. Recently, there has been a growing interest in tuning morphology by changing molecular structure [5], altering the composition of a binary gel $[6,7]$ and solvent $[8,9]$, and using ultrasound $[10,11]$, light $[12,13]$, and so on. For example, Saha et al. and coworkers reported for the first time the hierarchical tuning of one-dimensional morphology from helical bunched fibers to rods and hollow tubes, by changing the composition of riboflavin-melamine in a hydrogel system [2]. Zhu et al. and coworkers demonstrated the structural transition from organogels to flower-like microcrystals in dipeptide self-assembling system, which can be readily induced by using ethanol as a cosolvent [8]. Our previous work reported a photoinduced fiber-vesicle morphological transition in a chloroform gel of azophenyl hydrazide derivative [12]. However, full control over the morphologies of self-assembled structures for their implementation in special applications is still a great challenge.

Among the noncovalent interactions, hydrogen bonding is most commonly used to direct self-assembly process because of its strength, directionality, reversibility, and selectivity. Meanwhile, peptide, amino acid, amide, and urea groups have been widely employed as building blocks to afford supramolecular gels. Yang et al. and coworkers reported a hydrazide quinolinone-based quadruple hydrogen-bonded building block to gelate dichloromethane/hexane at concentration higher than $2 \mathrm{wt} \%$ [14]. Zhao et al. and coworkers reported a new series of highly stable hydrazide-based ADDA-DAAD heterodimers, which 


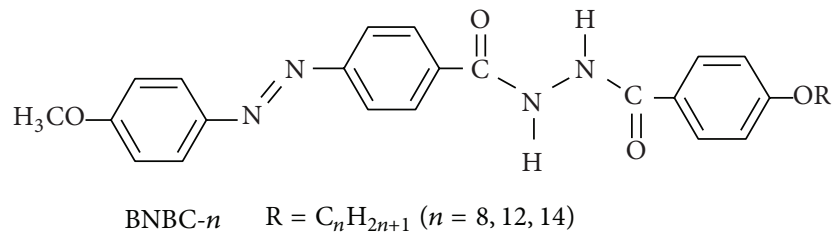

Scheme 1: The molecular structure of BNBC- $n$.

represented the first successful application of hydrazide derivatives in the hydrogen-mediated supramolecular systems with well-established structures [15]. In this work, we focus on the self-assembly structure of calamitic hydrazide derivatives, N-4-(alkoxyphenyl)- $\mathrm{N}^{\prime}-4$-[(4-methoxyphenyl)azophenyl] benzohydrazide (BNBC- $n, n=8,12,14$ ) (as shown in Scheme1), which had been synthesized and reported in our previous work [16]. The results indicate that the gelation property and the aggregation morphology of organogel strongly depend on the nature of gelling solvents and the length of terminal alkoxy chain. The possible mechanism for the formation of nanostructural aggregates is also discussed in this paper.

\section{Experimental Section}

Field emission scanning electron microscopy (FE-SEM) images were taken with a JSM-6700F apparatus. Samples for FE-SEM measurement were prepared by wiping a small amount of gel onto a silicon plate, followed by drying in a vacuum for $12 \mathrm{~h}$ at room temperature. FT-IR spectra were recorded with a Perkin-Elmer spectrometer (Spectrum One $\mathrm{B})$, in which the xerogels were prepared by freezing and pumping the organogel of BNBC- $n$ for $12 \mathrm{~h}$, and then pressed into a tablet with $\mathrm{KBr}$ for FT-IR measurement. UV-vis absorption spectra were obtained on a Shimadzu UV-2550 spectrometer.

Gelation Test. The weighted gelator was mixed in a cap sealed test tube $(3.5 \mathrm{~cm}$ (height) $\times 0.5 \mathrm{~cm}$ (radius)) with an appropriate amount of solvent, and the mixture was heated until the solid dissolved. The sample vial was cooled to $4^{\circ} \mathrm{C}$ and then turned upside down. When a clear or slightly opaque gel formed, the solvent therein was immobilized at this stage. Melting temperature $\left(T_{m}\right)$ was determined by the "falling drop" method [17]. An inverted gel was immersed in a water bath initially at or below room temperature and then was heated slowly up to the point at which the gel fell due to the force of gravity, that is, the $T_{m}$.

\section{Results and Discussion}

3.1. Molecular Design and Gelation Properties. The strategy of this work is to build up a system containing hydrogen bonding, $\pi-\pi$ interaction, and Van der Waals force to drive the self-assembly gelation. The mentioned above interactions play mutual balance to modulate the packing arrangement
TABLE 1: Gelation properties ${ }^{\mathrm{a}}$ of BNBC- $n, n=8,12,14$.

\begin{tabular}{lccccc}
\hline \multirow{2}{*}{ Solvent } & BNBC-8 & \multicolumn{2}{c}{ BNBC-12 } & \multicolumn{2}{c}{ BNBC-14 } \\
& State & State & CGC & State & CGC \\
\hline Cyclohexane & I & I & - & I & - \\
Toluene & P & G & $5.3 \times 10^{-3}$ & G & $6.7 \times 10^{-3}$ \\
Benzene & P & G & $8.2 \times 10^{-3}$ & G & $1.0 \times 10^{-2}$ \\
Chloroform & P & G & $1.3 \times 10^{-2}$ & G & $2.1 \times 10^{-2}$ \\
Acetone & P & G & $2.5 \times 10^{-2}$ & G & $4.8 \times 10^{-2}$ \\
Methanol & P & P & - & P & - \\
DMSO & P & S & - & S & - \\
\hline
\end{tabular}

${ }^{\mathrm{a}} \mathrm{G}$ : stable gel formed at room temperature; I: insoluble; P: precipitated; S: soluble; CGC: critical gelation concentration $(\mathrm{mol} / \mathrm{L})$, the minimum solute concentration necessary for gelation.

of molecules and eventually construct a particular superstructure. For this purpose, we had designed and synthesized the calamitic oxadiazole derivative BNBC- $n$, which contains hydrazide, azobenzene, and an alkyl chain with different length (as shown in Scheme 1). The numerous interactions in BNBC- $n$ should offer, at least to some extent, the possibility of controlling the aggregation morphology of organogel. A certain solubility of a gelator in solvents is a prerequisite for gelation, whereas microphase segregation from dissolved solvents will induce gelation, in which different types of intermolecular interactions underpin the self-assembly of gelators. The length of alkyl chain is expected to regulate the intermolecular interaction with solvent to achieve a suitable solubility in solvent and thus the self-assembled nanostructures of organogel. We have investigated the gelation ability of BNBC- $n$ in various organic solvents at room temperature, and the relevant minimum gel concentrations of BNBC- $n$ are summarized in Table 1.

The obtained results indicate that the BNBC- 8 bearing shorter terminal chain is difficult to dissolve in organic solvents with weak polarity, such as 1,2-dichloroethane and cyclohexane, but dissolves in medium and strong polarity such as benzene, chloroform, tetrahydrofuran, ethanol, and dimethylsulfoxide (DMSO) by heating and precipitates with cooling. In contrast, compounds BNBC-12 and BNBC-14 bearing longer terminal chain can form a stable gel in polar solvents such as aromatic solvents chloroform, while both of them dissolve in methanol by heating but precipitate with cooling as well. A possible reason for the precipitation from protonic solvents might be that the potential supramolecular aggregation through intermolecular hydrogen bonding is prevented in this case. In other words, the above gelation property in different solvents manifests that intermolecular hydrogen bonding between the hydrazide groups is the driving force for the gelation and subsequent nanostructure. Among these three calamitic hydrazide derivatives, BNBC12 bearing appropriate length of terminal chain shows the strongest gelation ability in toluene, and the critical gel concentration (CGC) can reach as low as $5.3 \times 10^{-3} \mathrm{~mol} / \mathrm{L}$, which can be considered as a supergelator. Moreover, the sol-gel transition of BNBC-12 and BNBC-14 is fully thermoreversible even after several cycles of heating and cooling. 


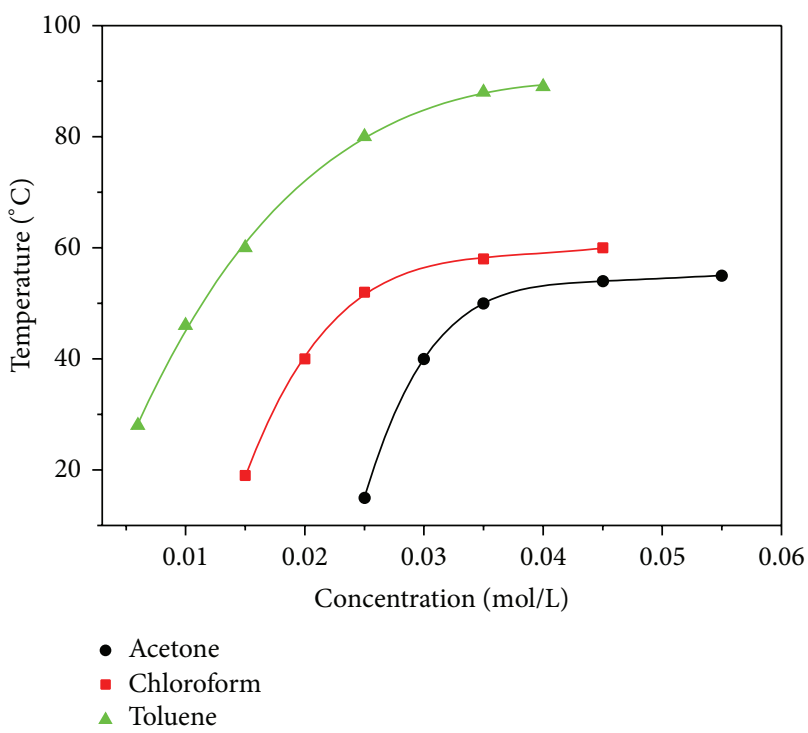

FIGURE 1: Concentration-dependent melting temperature $\left(T_{m}\right)$ of BNBC-12 gelating in toluene, chloroform, and acetone, respectively.

The organogels are remarkably stable and can be stored for months without showing any decomposition.

Figure 1 shows the gel-sol transition temperature $\left(T_{m}\right)$ of BNBC-12 gels in benzene, chloroform, and acetone as a function of concentration, respectively. The $T_{m}$ increases with the concentration until a plateau region is reached (denoted by a concentration-independent $T_{m}$ ), which is determined by the "falling drop" method [17]. With changing the solvent, the $T_{m}$ value in the "plateau region" decreases from $89^{\circ} \mathrm{C}$ (in toluene) to $60^{\circ} \mathrm{C}$ (in chloroform) and then to $55^{\circ} \mathrm{C}$ (in acetone). The most dramatic feature is that the morphologies of xerogels formed by BNBC-12 display a strong dependence on the nature of gelling solvent. From the summary listed in Table 1, it can be seen that the gelation ability of BNBC- $n$ manifests an apparent dependence on the length of terminal chain of BNBC- $n$ and intermolecular interaction with solvents, which will modify their self-assembled morphology structures as well.

3.2. Morphologies of the Xerogels. In order to investigate the aggregation morphology of organogel, the xerogels of BNBC- $n$ were prepared and subjected to scanning electron microscope (SEM). As shown in Figure 2(a), the organogel of BNBC-12 from acetone consists of flexible root-like fibers with the width of 100-500 nm and length of tens of micrometers. The more entangled and dense fiber morphology with the width of $30-50 \mathrm{~nm}$ is observed for xerogel BNBC-12 from toluene (Figure 2(b)), and these fibers further assemble into thick fiber bundles and then constitute a highly developed and entangled network, indicating that the interactions between individual fibers are stronger in toluene. Meanwhile, the gel of BNBC-12 in toluene exhibits better transparency. Interestingly, the morphologies of the BNBC-12 xerogel from chloroform show a quite different packing from that of other xerogels. As shown in Figure 2(c), the xerogel of
BNBC-12 from toluene consists of coral-shaped aggregation morphology and reveals 3D cross-linking network structure. From the zoom-in of top right corner in Figure 2(c) (as shown in Figure 2(d)), it can be seen that the coralloid aggregation structure is composed by hillocks with the size of $50 \mathrm{~nm}$ in diameter and $50-200 \mathrm{~nm}$ in length. As to the xerogel of BNBC-14 from toluene (Figure 2(f)), the morphology image exhibits straight and dense fibrous aggregates with the diameter of $30-80 \mathrm{~nm}$ and tens of micrometers in length. The aggregate structure of BNBC-14 xerogel from acetone (Figure 2(e)) is composed of flat ribbons with the width ranging from $1 \mu \mathrm{m}$ to $5 \mu \mathrm{m}$, and some ribbons bent to form hollow tube as shown in the inset of Figure 2(e). The observed morphology results indicate the gelation ability of BNBC12 in different organic solvents is stronger than that of BNBC-14. Correspondingly, the assembled fiber or ribbon of BNBC-12 would capture more solvent molecules and would demonstrate a lower CGC value as well.

On the basis of the above results, it can be concluded that the morphology of the BNBC- $n$ xerogels strongly depends on the nature of gelling solvents. These observations of tunable organogel structure are consistent with the CGC in different solvents shown in Table 1 for BNBC-12 and BNBC-14. The formation of elongated fiber-like and coral-shaped aggregates indicates that the self-assembly of BNBC- $n$ is driven by strong intermolecular interactions.

3.3. The Interactions in the Gels. To investigate hydrogen bonding and alkyl chain conformations in the gelation process, the Fourier transform infrared (FT-IR) measurements on the xerogels of BNBC-12 from toluene, chloroform, and acetone were carried out, respectively. FT-IR spectroscopy of BNBC-12 xerogel from toluene (Figure 3(a)) shows that the N-H stretching vibrations are at around $3220 \mathrm{~cm}^{-1}$ and amide I at around $1641 \mathrm{~cm}^{-1}$ and $1679 \mathrm{~cm}^{-1}$, respectively, indicating that the $\mathrm{N}-\mathrm{H}$ groups are associated with $\mathrm{C}=\mathrm{O}$ groups via $\mathrm{N}-\mathrm{H} \cdots \mathrm{O}=\mathrm{C}$ hydrogen bonding in the xerogel $[18$, 19]. For the acetone xerogel (Figure 3(c)), the $\mathrm{N}-\mathrm{H}$ stretching vibrations locate at $3230 \mathrm{~cm}^{-1}$, and the peak slightly shifts to higher wavenumbers along with a shift of amide I bands to $1683 \mathrm{~cm}^{-1}$ and $1645 \mathrm{~cm}^{-1}$, respectively. Thus, it can be concluded that intermolecular hydrogen bonding exists in BNBC-12 xerogel from acetone, though it is somewhat weaker compared with that in the corresponding xerogel from toluene. On the other hand, the $v_{s}\left(\mathrm{CH}_{2}\right)$ and $v_{\mathrm{as}}\left(\mathrm{CH}_{2}\right)$ in the xerogel of BNBC-12 appear at around $2855 \mathrm{~cm}^{-1}$ and $2925 \mathrm{~cm}^{-1}$, respectively, implying that the alkyl chains are closely packed to form quasi-crystalline domain [20].

Electronic UV-vis absorption spectra of the gels were studied to obtain information about the aggregated state of azobenzene on the molecular scale. As shown in Figure 4, the absorption spectra of BNBC-12 manifest a slight but detectable dependence on concentration in acetone. With a dilute $\left(1 \times 10^{-5} \mathrm{M}\right)$ solution of BNBC-12 in acetone, the $\pi-\pi^{*}$ absorption maximum of the azobenzene group of BNBC12 (Figure 4) locates at $351 \mathrm{~nm}$. With the concentration increasing from $1 \times 10^{-5} \mathrm{M}$ to $1 \times 10^{-3} \mathrm{M}$, the absorption maximum is slightly red-shifted from $351 \mathrm{~nm}$ to $357 \mathrm{~nm}$, indicating 


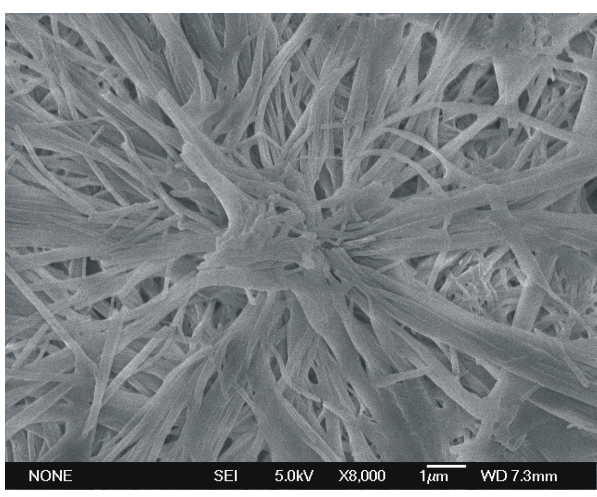

(a)

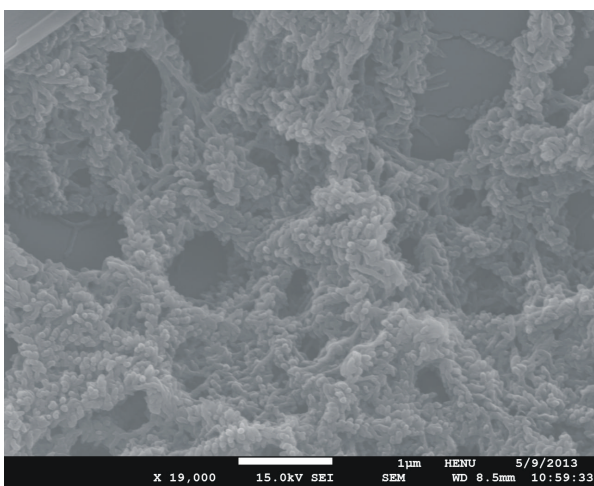

(c)

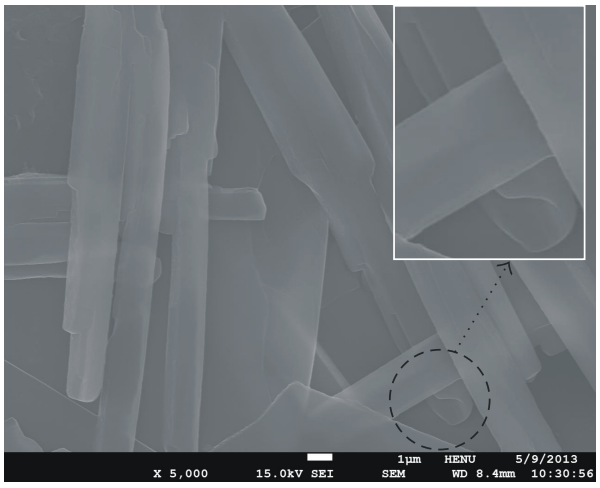

(e)

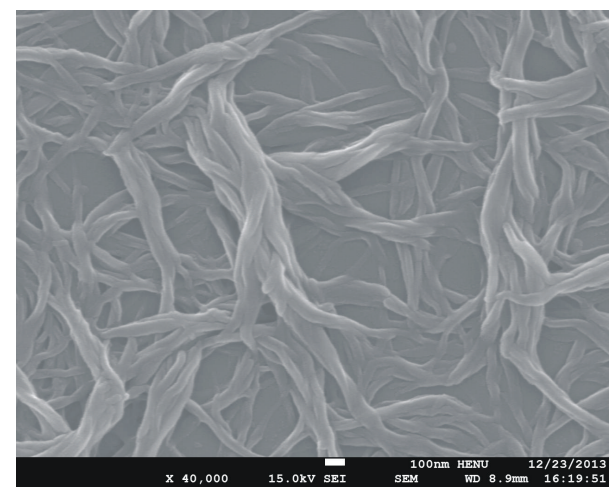

(b)

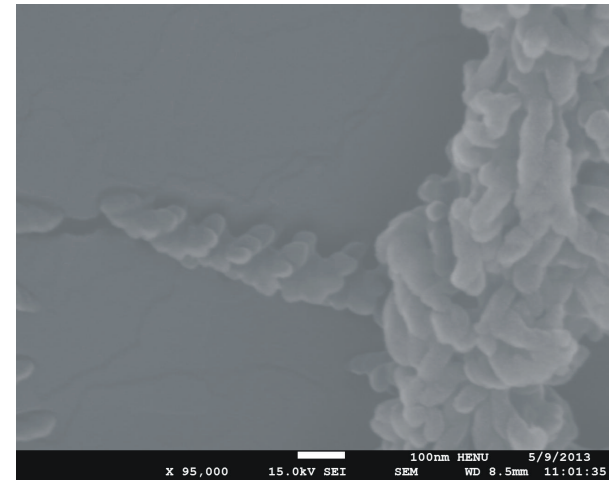

(d)

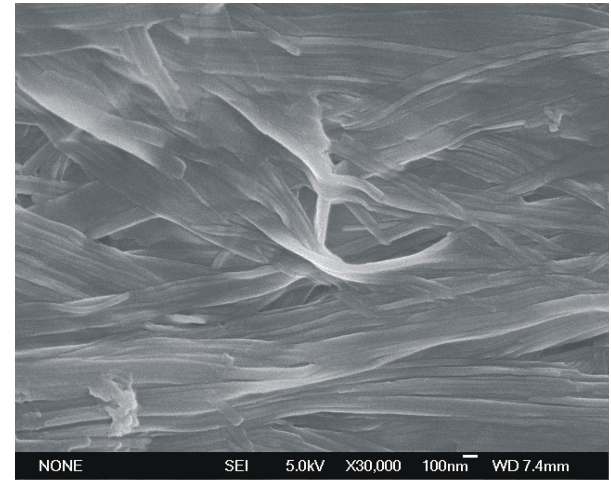

(f)

FIGURE 2: SEM images of (a) BNBC-12 xerogels from acetone $\left(3.0 \times 10^{-2} \mathrm{M}\right)$, (b) BNBC-12 xerogels from toluene $\left(5.8 \times 10^{-3} \mathrm{M}\right)$, $(\mathrm{c}, \mathrm{d}) \mathrm{BNBC}$ 12 xerogels from chloroform $\left(3.0 \times 10^{-2} \mathrm{M}\right)$, (e) BNBC-14 xerogels from acetone $\left(5.0 \times 10^{-2} \mathrm{M}\right)$, and (f) BNBC-14 xerogels from toluene $\left(8.0 \times 10^{-3} \mathrm{M}\right)$.

that the azobenzene units are arranged into J-type aggregates through $\pi-\pi$ interactions in gels [21]. Similar results were observed for BNBC-14 in acetone.

The self-assembled gelation is a complex process in which several noncovalent interactions are involved in the formation of aggregation structure. The above spectroscopic results with respect to the gelation ability and tunable morphology of BNBC-12 and BNBC-14 indicate that the cooperation of hydrogen bonds, $\pi-\pi$ interaction, and Van der Waals force plays an important role in self-assembly. The gelation of BNBC- $n$ provides an easy method for preparation of multidimensional structure and manipulation of morphology ranging from ribbons, hollow tubes, fibers to even $3 \mathrm{D}$ net-like structure in different solvents.

\section{Conclusions}

We have studied the gelation ability and morphology of a series of LMOG (BNBC- $n, n=8,12,14$ ) containing hydrazide, azobenzene, and alkyl chain with different length. BNBC-8 demonstrates a nonorganogel compound in any solvents or at different temperature. The organogelators BNBC-12 and BNBC-14 bearing longer terminal chains show strong gelation ability in organic solvents, such as toluene, 


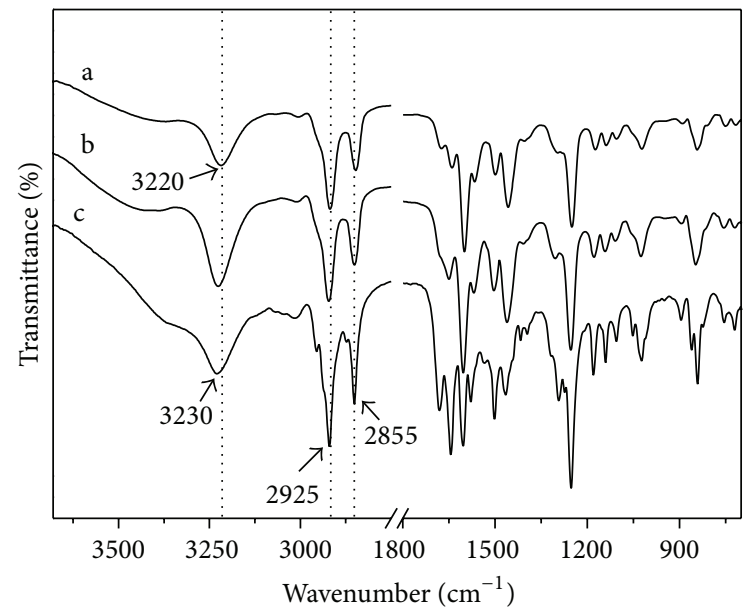

FigURE 3: FT-IR spectra of BNBC-12 xerogel from (a) toluene, (b) chloroform, and (c) acetone.

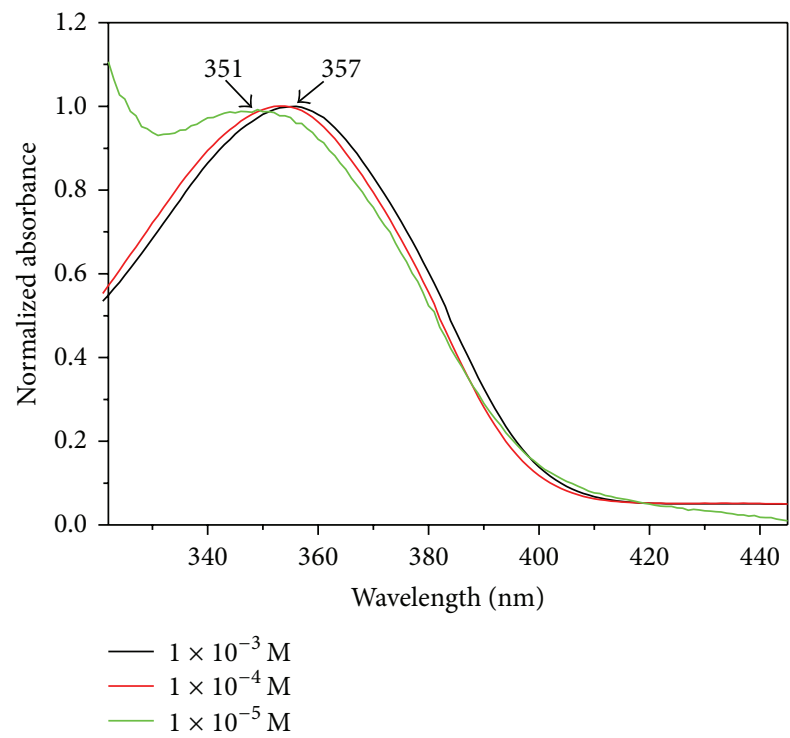

FIGURE 4: Normalized UV-vis absorption spectra of BNBC-12 in acetone at different concentrations.

chloroform and acetone, and so forth. The minimum gel concentration of BNBC-12 in toluene is as low as $5.3 \times 10^{-3} \mathrm{M}$, which can be considered as a supergelator. It also has been demonstrated that the morphology of the xerogels strongly depends on the nature of gelling solvents, and the selfassembled nanostructure is tunable by the intermolecular interactions between molecule unit with different length of terminal chain and solvents. Based on these observations, the cooperation of hydrogen bonds, $\pi-\pi$ interaction, and Van der Waals force might be crucially involved in the process of self-assembly. The unique and tunable aggregation morphology could be applied to surface modification such as superhydrophobicity and distinguish the obtained organogels as a novel class of functional materials.

\section{Conflict of Interests}

The authors declare that there is no conflict of interests regarding the publication of this paper.

\section{Acknowledgments}

The authors are grateful to the National Science Foundation Committee of China (Project nos. 21173068 and 21373077) and the Program for Innovative Research Team in University of Henan Province (no. 13IRTSTHN017) for their financial support of this work.

\section{References}

[1] D. J. Abdallah and R. G. Weiss, "Organogels and low molecular mass organic gelators," Advanced Materials, vol. 12, no. 17, pp. 1237-1347, 2000.

[2] A. Saha, S. Manna, and A. K. Nandi, "Hierarchical tuning of 1$\mathrm{D}$ macro morphology by changing the composition of a binary hydrogel and its influence on the photoluminescence property," Chemical Communications, no. 32, pp. 3732-3734, 2008.

[3] J. H. van Esch and B. L. Feringa, "Functional functional materials based on self-assembling organogels: from serendipity towards design," Angewandte Chemie International Edition, vol. 39, no. 13, pp. 2263-2266, 2000.

[4] J. M. Lehn, Supramolecular Chemistry: Concepts and Perspectives, Wiley-VCH, Weinheim, Germany, 1995.

[5] S. Qu, L. Zhao, Z. Yu et al., "Nanoparticles, helical fibers, and nanoribbons of an achiral twin-tapered bi-1,3,4-oxadiazole derivative with strong fluorescence," Langmuir, vol. 25, no. 3, pp. 1713-1717, 2009.

[6] K. Jang, J. M. Kinyanjui, D. W. Hatchett, and D.-C. Lee, "Morphological control of one-dimensional nanostructures of Tshaped asymmetric bisphenazine," Chemistry of Materials, vol. 21, no. 10, pp. 2070-2076, 2009.

[7] B. Roy, P. Bairi, and A. K. Nandi, "Supramolecular assembly of melamine and its derivatives: nanostructures to functional materials," RSC Advances, vol. 4, no. 4, pp. 1708-1734, 2014.

[8] P. Zhu, X. Yan, Y. Su, Y. Yang, and J. Li, "Solvent-induced structural transition of self-assembled dipeptide: from organogels to microcrystals," Chemistry - A European Journal, vol. 16, no. 10, pp. 3176-3183, 2010.

[9] W. Cai, G.-T. Wang, Y.-X. Xu, X.-K. Jiang, and Z.-T. Li, "Vesicles and organogels from foldamers: a solvent-modulated selfassembling process," Journal of the American Chemical Society, vol. 130, no. 22, pp. 6936-6937, 2008.

[10] T. Naota and H. Koori, "Molecules that assemble by sound: an application to the instant gelation of stable organic fluids," Journal of the American Chemical Society, vol. 127, no. 26, pp. 9324-9325, 2005.

[11] R.-Y. Wang, X.-Y. Liu, and J.-L. Li, "Engineering molecular selfassembled fibrillar networks by ultrasound," Crystal Growth \& Design, vol. 9, no. 7, pp. 3286-3291, 2009.

[12] X. Ran, H. Wang, P. Zhang et al., "Photo-induced fiber-vesicle morphological change in an organogel based on an azophenyl hydrazide derivative," Soft Matter, vol. 7, no. 18, pp. 8561-8566, 2011.

[13] L. Frkanec, M. Jokić, J. Makarević, K. Wolsperger, and M. Žinić, "Bis(PheOH) maleic acid amide-fumaric acid amide photoizomerization induces microsphere-to-gel fiber morphological transition: the photoinduced gelation system," Journal of the American Chemical Society, vol. 124, no. 33, pp. 9716-9717, 2002. 
[14] Y. Yang, H.-J. Yan, C.-F. Chen, and L.-J. Wan, "Quadruply hydrogen-bonded building block from hydrazide-quinolinone motif and gelation ability of its analogous oxalic monoestermonoamide derivative," Organic Letters, vol. 9, no. 24, pp. 49914994, 2007.

[15] X. Zhao, X.-Z. Wang, X.-K. Jiang, Y.-Q. Chen, Z.-T. Li, and G.J. Chen, "Hydrazide-based quadruply hydrogen-bonded heterodimers. Structure, assembling selectivity, and supramolecular substitution," Journal of the American Chemical Society, vol. 125, no. 49, pp. 15128-15139, 2003.

[16] X. Ran, H. Wang, P. Zhang et al., "Anticlinic smectic phase formed by calamitic hydrazide derivatives with terminal hydroxyl group," Liquid Crystals, vol. 38, no. 10, pp. 1227-1237, 2011.

[17] D. J. Abdallah and R. G. Weiss, "n-Alkanes gel n-alkanes (and many other organic liquids)," Langmuir, vol. 16, no. 2, pp. 352$355,2000$.

[18] S. Qu, F. Li, H. Wang et al., "Twin-tapered molecules containing Bi-dihydrazine units: self-assembly through intermolecular quadruple hydrogen bonding and liquid crystalline behavior," Chemistry of Materials, vol. 19, no. 20, pp. 4839-4846, 2007.

[19] H. Shen, K.-U. Jeong, H. Xiong et al., "Phase behaviors and supra-molecular structures of a series of symmetrically tapered bisamides," Soft Matter, vol. 2, no. 3, pp. 232-242, 2006.

[20] N. V. Venkataraman and S. Vasudevan, "Conformation of methylene chains in an intercalated surfactant bilayer," Journal of Physical Chemistry B, vol. 105, no. 9, pp. 1805-1812, 2001.

[21] M. Shimomura and T. Kunitake, "Fluorescence and photoisomerization of azobenzene-containing bilayer membranes," Journal of the American Chemical Society, vol. 109, no. 17, pp. 5175-5183, 1987. 

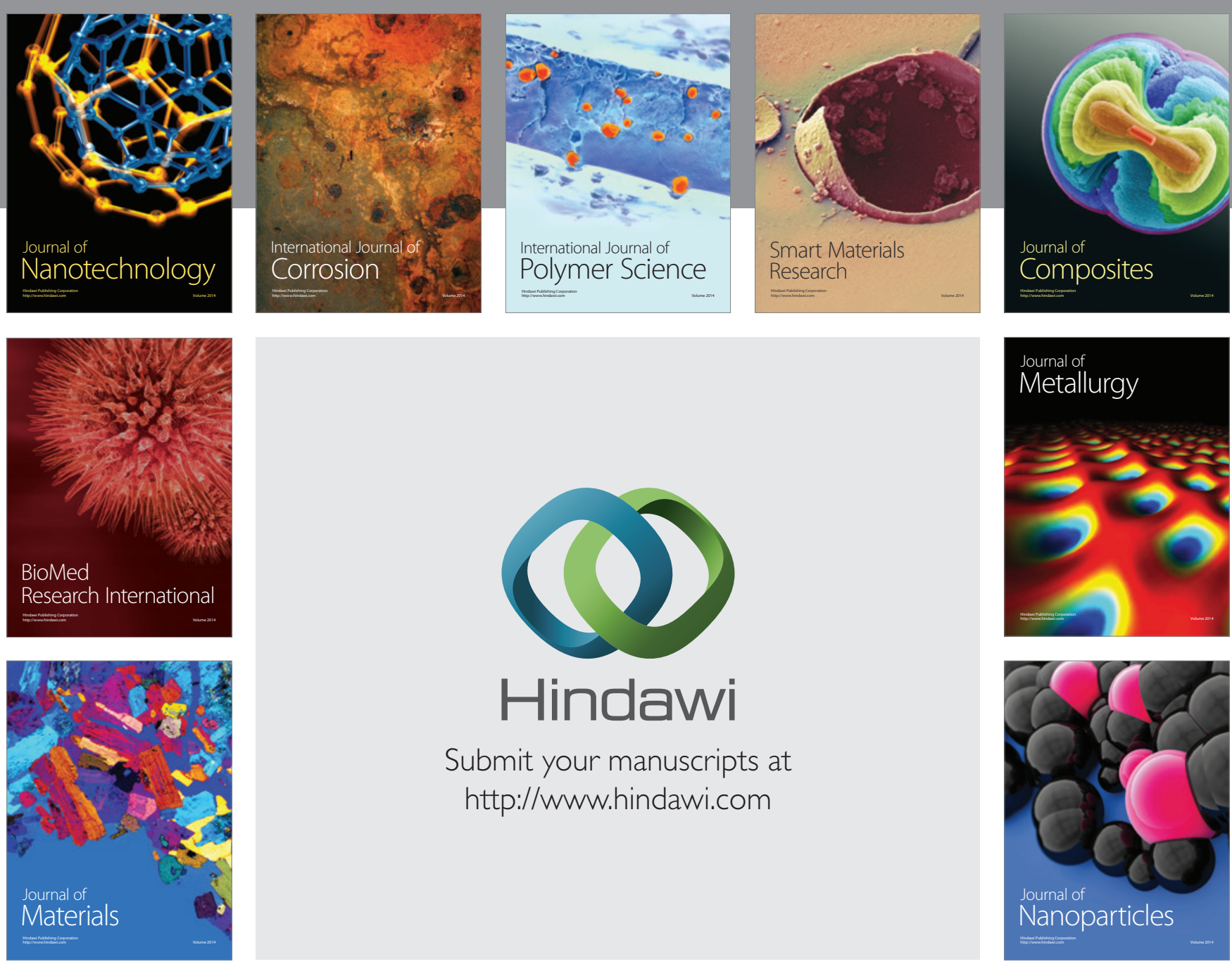

Submit your manuscripts at http://www.hindawi.com
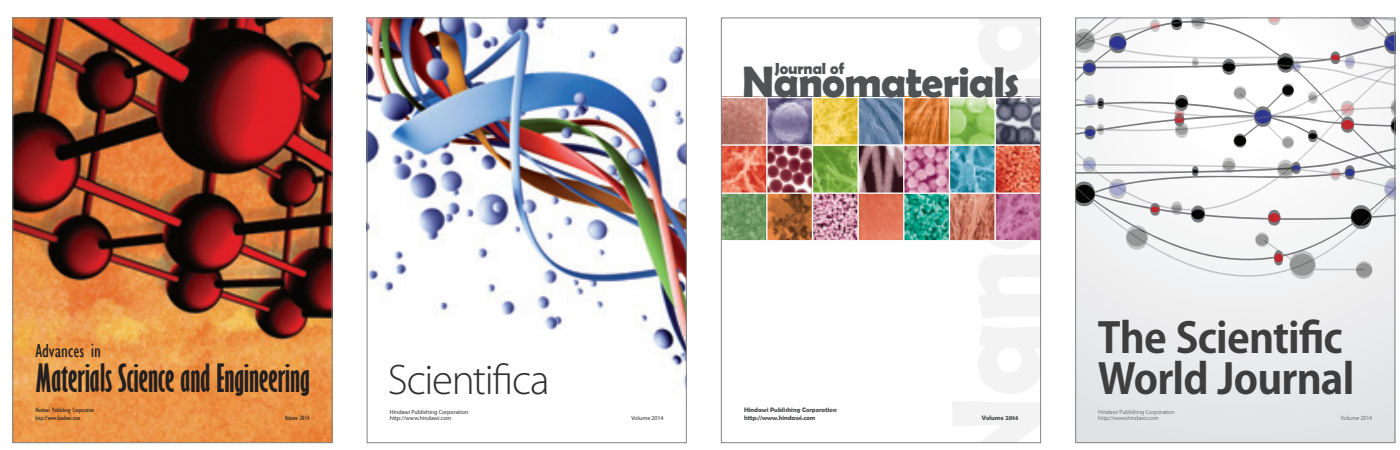

\section{The Scientific World Journal}
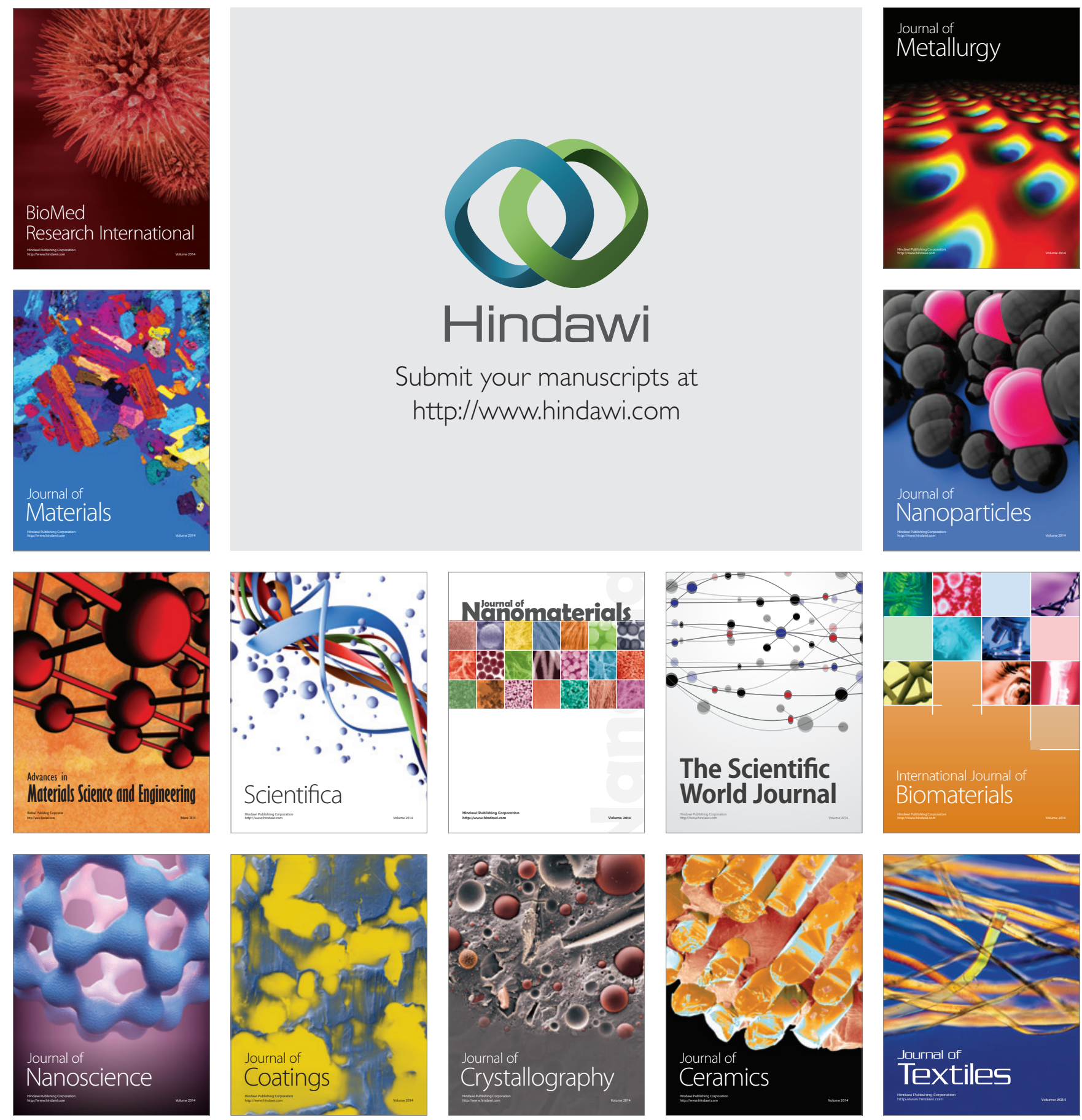